\title{
The Drury-Arveson Space on the Siegel Upper Half-space and a von Neumann Type Inequality
}

\author{
Nicola Arcozzi@, Nikolaos Chalmoukis@, Alessandro Monguzzi®®, \\ Marco M. Peloso® and Maura Salvatori®
}

\begin{abstract}
In this work we study what we call Siegel-dissipative vector of commuting operators $\left(A_{1}, \ldots, A_{d+1}\right)$ on a Hilbert space $\mathcal{H}$ and we obtain a von Neumann type inequality which involves the Drury-Arveson space $D A$ on the Siegel upper half-space $\mathcal{U}$. The operator $A_{d+1}$ is allowed to be unbounded and it is the infinitesimal generator of a contraction semigroup $\left\{e^{-i \tau A_{d+1}}\right\}_{\tau<0}$. We then study the operator $e^{-i \tau A_{d+1}} A^{\alpha}$ where $A^{\alpha}=A_{1}^{\alpha_{1}} \cdots A_{d}^{\alpha_{d}}$ for $\alpha \in \mathbb{N}_{0}^{d}$ and prove that can be studied by means of model operators on a weighted $L^{2}$ space. To prove our results we obtain a Paley-Wiener type theorem for $D A$ and we investigate some multiplier operators on $D A$ as well.
\end{abstract}

Mathematics Subject Classification. 47A13, 47B37, 46E22.

Keywords. Siegel upper half-space, Holomorphic function spaces, Drury - Arveson, Von Neumann inequality.

\section{Introduction}

The Drury-Arveson space $D A\left(\mathbb{B}^{d+1}\right)$ on the unit ball of $\mathbb{C}^{d+1}$ is a renowned Hilbert space of holomorphic functions endowed with the inner product

$$
\left\langle\sum_{\alpha \in \mathbb{N}_{0}^{d+1}} b_{\alpha} z^{\alpha}, \sum_{\alpha \in \mathbb{N}_{0}^{d+1}} c_{\alpha} z^{\alpha}\right\rangle_{D A\left(\mathbb{B}^{d+1}\right)}:=\sum_{\alpha \in \mathbb{N}_{0}^{d+1}} b_{\alpha} \overline{c_{\alpha}} \frac{\alpha !}{|\alpha| !} .
$$

We refer the reader to $[4$, Theorem 6.1$]$ for an exact integral representation of such inner product. The space $D A\left(\mathbb{B}^{d+1}\right)$ is a reproducing kernel Hilbert space with kernel $K(z, w)=(1-z \cdot \bar{w})^{-1}$ and may be considered the natural

All the authors are members of INdAM. The first and third author are partially supported by the Progetto GNAMPA 2020 Alla frontiera tra l'analisi complessa in più variabili e l'analisi armonica. The fourth and fifth author are partially supported by the Progetto GNAMPA 2020 Fractional Laplacians and subLaplacians on Lie groups and trees. 
multi-dimensional version of the Hardy space on the unit disc $H^{2}(\mathbb{D})$; one of the main reasons being that $D A\left(\mathbb{B}^{d+1}\right)$ plays the same role as $H^{2}(\mathbb{D})$ in a multi-dimensional version of the famous von Neumann Inequality.

Theorem 1.1. (Drury [9]) Let $\mathcal{H}$ be a Hilbert space and consider the $(d+1)$ tuple $T=\left(T_{1}, \ldots, T_{d+1}\right)$ of linear operators on $\mathcal{H}$ satisfying

(i) $T_{j} T_{k}-T_{k} T_{j}=0$ for all $j, k=1, \ldots, d+1$;

(ii) $\sum_{j=1}^{d+1}\left\|T_{j} v\right\|_{\mathcal{H}}^{2} \leq\|v\|_{\mathcal{H}}^{2}$ for all $v \in \mathcal{H}$.

Let $p(z)=p\left(z_{1}, \ldots, z_{d+1}\right)$ be a complex polynomial. Then,

$$
\|p(T)\|_{\mathcal{B}(\mathcal{H})} \leq\|p\|_{\mathcal{M}\left(D A\left(\mathbb{B}^{d+1}\right)\right)}
$$

where $\|\cdot\|_{\mathcal{B}(\mathcal{H})}$ and $\|\cdot\|_{\mathcal{M}\left(D A\left(\mathbb{B}^{d+1}\right)\right)}$ denote the norm of bounded linear operators on $\mathcal{H}$ and the multiplier operator norm on the Drury-Arveson space on the unit ball respectively.

In the case $d=0$ Drury's result reduces to the classical von Neumann Inequality $([22])$ and the multiplier operator norm on $H^{2}(\mathbb{D})$ takes the place of $\|\cdot\|_{\mathcal{M}\left(D A\left(\mathbb{B}^{d+1}\right)\right)}$. Namely, given a Hilbert space $\mathcal{H}$ and $T: \mathcal{H} \rightarrow \mathcal{H}$ a contraction, i.e., $\|T\|_{\mathcal{B}(\mathcal{H})} \leq 1$, the von Neumann Inequality states that, for any polynomial $p(z)$,

$$
\|p(T)\|_{\mathcal{B}(\mathcal{H})} \leq\|p\|_{\mathcal{M}\left(H^{2}(\mathbb{D})\right)}=\|p\|_{\infty} .
$$

It is possible to prove an analogous result modeled on the upper-half plane, which is the unbounded biholomorphic realization of the unit disk $\mathbb{D}$ via the Cayley transform. Let $A$ be any bounded dissipative operator on a Hilbert space $\mathcal{H}$, that is, such that $\frac{1}{i}\left(A-A^{*}\right) \geq 0$, then

$$
\|f(A)\|_{\mathcal{B}(\mathcal{H})} \leq \sup _{\operatorname{Im} z>0}|f(z)|
$$

for any rational function $f$ which is bounded on the upper half-plane, see $[23]$.

The main goal of this paper is to prove a version of Theorem 1.1 on an unbounded biholomorphic realization of the unit ball $\mathbb{B}^{d+1}$ in $\mathbb{C}^{d+1}$, that is, the Siegel upper-half space $\mathcal{U}$,

$$
\mathcal{U}=\left\{\left(\zeta, \zeta_{d+1}\right): \mathbb{C}^{d+1}: \operatorname{Im} \zeta_{d+1}>\frac{1}{4}|\zeta|^{2}\right\} .
$$

The biholomorphism between the ball and the Siegel half-space is given by the multi-dimensional Cayley transform $\mathcal{C}: \mathbb{B}^{d+1} \rightarrow \mathcal{U}$,

$$
\mathcal{C}\left(\omega, \omega_{d+1}\right)=\left(\frac{2 \omega}{1-\omega_{d+1}}, i \frac{1+\omega_{d+1}}{1-\omega_{d+1}}\right) .
$$

The Drury-Arveson space on $\mathcal{U}$, that we shall simply denote by $D A$, was studied in [6] and [4], where an integral expression for the norm was also obtained. Let $\rho\left(\zeta, \zeta_{d+1}\right)=\operatorname{Im} \zeta_{d+1}-\frac{1}{4}|\zeta|^{2}$ and let $n$ be an integer such that $n>d / 2$. Then, we define the space $D A_{(n)}$ as

$$
\begin{aligned}
& D A_{(n)}=\left\{F \in \operatorname{Hol}(\mathcal{U}):(\mathrm{i}) \lim _{|\zeta| \leq R, \operatorname{Im} \zeta_{d+1} \rightarrow+\infty} F\left(\zeta, \zeta_{d+1}\right)=0\right. \\
& \text { (ii) } \left.\int_{\mathcal{U}}\left|\rho^{n}\left(\zeta, \zeta_{d+1}\right) \partial_{\zeta_{d+1}}^{n} F\left(\zeta, \zeta_{d+1}\right)\right|^{2} \rho^{-d-1}\left(\zeta, \zeta_{d+1}\right) d \zeta d \zeta_{d+1}<+\infty\right\} .
\end{aligned}
$$


The space $D A_{(n)}$ does not actually depend on $n$, so that we simply write $D A$ in place of $D A_{(n)}$. We refer the reader to Sect. 3 for this and other properties of the space $D A$.

We now describe the operator-theorical condition we shall work with.

Definition 1.2. Let $\mathcal{H}$ be a Hilbert space and let $\left(A_{1}, \ldots, A_{d+1}\right)$ be a vector of operators on $\mathcal{H}$ and let $r\left(i A_{d+1}\right)$ be the resolvent set of $i A_{d+1}$. We say that $\left(A_{1}, \ldots, A_{d+1}\right)$ is a Siegel-dissipative vector of commuting operators if:

(i) the operators $A_{1}, \ldots A_{d}$ are bounded and $(0,+\infty) \subseteq r\left(i A_{d+1}\right)$;

(ii) the operators $A_{1}, \ldots A_{d}$ commute with each other and they strongly commute with $A_{d+1}$;

(iii) the following condition holds: ${ }^{1}$

$$
\operatorname{Im}\left\langle A_{d+1} v, v\right\rangle_{\mathcal{H}} \geq \frac{1}{4} \sum_{i=1}^{d}\left\|A_{i} v\right\|_{\mathcal{H}}^{2}, \quad \forall v \in \operatorname{Dom}\left(A_{d+1}\right) .
$$

Given two operators $U$ and $T$ on $\mathcal{H}, U$ densely defined and closed, $T$ bounded, we say that $U$ and $T$ strongly commute if $U T$ is an extension of $T U$. In other words $T(\mathcal{H}) \subseteq \operatorname{Dom}(U)$ and $T U v=U T v$ for all $v \in \operatorname{Dom}(U)$.

We point out that from the conditions above and the Lumer-Philips Theorem (Theorem 2.5) it follows that $i A_{d+1}$ is the infinitesimal generator of a (unique) semigroup of contractions $\left\{e^{-i \tau A_{d+1}}\right\}_{\tau \leq 0}$ that commutes with all the $A_{j}$ 's, see Lemma 5.1. Our von Neumann type inequality reads as follows.

Theorem 1.3. Let $\mathcal{H}$ be a Hilbert space and let $\left(A_{1}, \ldots, A_{d+1}\right)$ be a Siegeldissipative vector of commuting operators. For any $\tau_{j}<0, j=1, \ldots, d$, set $M_{j}=e^{-i \tau_{j} A_{d+1}} A_{j}$ and $m_{j}\left(\zeta, \zeta_{d+1}\right)=e^{-i \tau_{j} \zeta_{d+1}} \zeta_{j}$. Let $p$ denote any polynomial in $d$ variables. Then we have that $m_{j} \in \mathcal{M}(D A)$ for $j=1, \ldots, d$, and

$$
\left\|p\left(M_{1}, \ldots, M_{d}\right)\right\|_{\mathcal{B}(\mathcal{H})} \leq\left\|p\left(m_{1}, \ldots, m_{d}\right)\right\|_{\mathcal{M}(D A)},
$$

where $\mathcal{M}(D A)$ is the space of pointwise multipliers on the Drury-Arveson space $D A$.

A few remarks are now in order. The space $D A$ is a reproducing kernel Hilbert space, and, with respect to the $D A_{(n)}$-norm, its reproducing kernel is

$$
K_{D A}\left(\left(\omega, \omega_{d+1}\right),\left(\zeta, \zeta_{d+1)}\right)=\gamma_{d, n}\left(\frac{\omega_{d+1}-\bar{\zeta}_{d+1}}{2 i}-\frac{1}{4} \omega \cdot \bar{\zeta}\right)^{-1},\right.
$$

where $\gamma_{d, n}=\frac{4^{n}}{(4 \pi)^{d+1} \Gamma(2 n-d)}$. Hence, notice that the condition (1.4) is modelled after the reproducing kernel of $D A$. In general, it is a well established phenomenon that von Neumann type inequalities hold as long as one can suitably interpret the condition $K^{-1}(A, A) \geq 0$, where $K$ is the reproducing kernel of a space of holomorphic functions in some domain in $\mathbb{C}^{d}$. A precise formulation of this result is stated and proved in [2]. There the authors use

\footnotetext{
${ }^{1}$ The multiplicative constant $\frac{1}{4}$ in (1.4) appears because of the definition of $\mathcal{U}$ we use. The presence of this constant might be unpleasant but we keep this definition to be consistent with the definition in [4].
} 
the Dunford-Riesz functional calculus to define $K^{-1}(A, A)$. This is possible since they are working with bounded operators with some additional assumptions on their spectrum. Furthermore, they assume that the multiplication by any coordinate function is a continuous (bounded) operation. Our setting differs in these two latter aspects. The last operator $A_{d+1}$ is allowed to be unbounded and also the multiplication by any coordinate is an unbounded operation in $D A$.

We also mention that, in the case when all the operators are bounded, a proof of the von Neumann type inequality (1.5) could probably be obtained by means of the Cayley transform, the classical inequality of Drury on the unit ball and some classical results in operator theory as it was pointed out to us by M. Hartz in a private communication. Nevertheless, in the footsteps of Drury's proof, we prefer to use a direct approach, without relying on the known results on the unit ball, for two main reasons. First, we have greater generality by allowing one operator to be unbounded. Second, and more importantly, we develop some machinery we believe it is interesting in its own right and has the potential to be used in the context of the theory of shift-invariant subspaces of $D A$, as in the spirit of Buerling-Lax's Theorem [12], in the Siegel half-space setting.

In order to follow such a plan, we further investigate some operatortheorical properties of $D A$. On the space $\mathbb{N}_{0}^{d} \times \mathbb{R}_{-}$we consider the measure

$$
d \mu(\alpha, \lambda)=\alpha !\left(\frac{2}{|\lambda|}\right)^{|\alpha|}|\lambda|^{2 d} d \alpha d \lambda
$$

with $d \lambda$ denoting the Lebesgue measure on $\mathbb{R}_{-}$and $d \alpha$ the counting measure on $\mathbb{N}_{0}^{d}$.

Theorem 1.4. The map $\mathscr{S}: L^{2}\left(\mathbb{N}_{0}^{d} \times \mathbb{R}_{-}, d \mu\right) \rightarrow D A$ defined as

$$
(\mathscr{S} \varphi)\left(\zeta, \zeta_{n+1}\right)=\frac{1}{(2 \pi)^{d+1}} \int_{\mathbb{N}_{0}^{d} \times \mathbb{R}_{-}} \zeta^{\alpha} e^{-i \lambda \zeta_{d+1}} \overline{\varphi(\alpha, \lambda)}|\lambda|^{d} d \alpha d \lambda
$$

is a conjugate linear surjective isometry. The inverse map $\mathscr{S}^{-1}$ is explicitly given in (3.1).

If for some $\varepsilon>0$ the $(d+1)$-tuple $\left(A_{1}, \ldots, A_{d}, A_{d+1}-i \varepsilon \mathrm{Id}\right)$ is Siegeldissipative, we say that $\left(A_{1}, \ldots, A_{d+1}\right)$ is strongly Siegel-dissipative. In this case, for $v, v^{\prime} \in \operatorname{Dom}\left(A_{d+1}\right)$ consider the inner product

$$
\left\langle v, v^{\prime}\right\rangle_{\Delta}:=\frac{1}{2 i}\left(\left\langle A_{d+1} v, v^{\prime}\right\rangle_{\mathcal{H}}-\left\langle v, A_{d+1} v^{\prime}\right\rangle_{\mathcal{H}}\right)-\frac{1}{4} \sum_{i=1}^{d}\left\langle v, v^{\prime}\right\rangle_{\mathcal{H}}
$$

so that

$$
\|v\|_{\Delta}^{2}=\operatorname{Im}\left\langle A_{d+1} v, v\right\rangle_{\mathcal{H}}-\frac{1}{4} \sum_{i=1}^{d}\left\|A_{i} v\right\|_{\mathcal{H}}^{2} \geq \varepsilon\|v\|_{\mathcal{H}}^{2}, \quad \forall v \in \operatorname{Dom}\left(A_{d+1}\right),
$$


which is a stronger condition than (1.4). If $\mathcal{H}_{\Delta}=\left(\right.$ Dom $\left.A_{d+1},\|\cdot\|_{\Delta}\right)$ we define

$$
\begin{aligned}
& \mathcal{L}^{2}(\Delta):=L^{2}\left(\mathbb{N}_{0}^{d} \times \mathbb{R}_{-}, d \mu ; \mathcal{H}_{\Delta}\right) \\
& \quad=\left\{g: \mathbb{N}_{0}^{d} \times \mathbb{R}_{-} \rightarrow \operatorname{Dom} A_{d+1}: \sum_{\alpha \in \mathbb{N}_{0}^{d}} \int_{-\infty}^{0}\|g(\alpha, \lambda)\|_{\Delta}^{2} d \mu(\alpha, \lambda)<+\infty\right\} .
\end{aligned}
$$

With our assumptions it follows that $\mathcal{L}^{2}(\Delta)$ is a pre-Hilbert space. Before stating our result on $d+1$-tuples of operators we also need to study some weighted shift operators on $L^{2}\left(\mathbb{N}_{0}^{d} \times \mathbb{R}_{-}, d \mu\right)$, which correspond to some multiplier operators on $D A$ (see Sect. 4). If $\alpha, \gamma \in \mathbb{N}_{0}^{d}$ we write $\alpha \geq \gamma$ meaning that $\alpha_{i} \geq \gamma_{i}$ for all $i=1, \ldots, d$. Then, we prove the following.

Theorem 1.5. Let $\gamma \in \mathbb{N}_{0}^{d}$ and $\tau<0$. Then, the operator

$$
\left(S_{\gamma, \tau} \varphi\right)(\alpha, \lambda)= \begin{cases}\frac{|\lambda-\tau|^{d}}{|\lambda|^{d}} \varphi(\alpha-\gamma, \lambda-\tau) & \text { if } \lambda<\tau \wedge \alpha \geq \gamma \\ 0 & \text { otherwise, }\end{cases}
$$

extends to a bounded linear operator on $L^{2}\left(\mathbb{N}_{0}^{d} \times \mathbb{R}_{-}, d \mu\right)$ unitarily equivalent to the multiplier operator on DA with multiplier $\zeta^{\gamma} e^{-i \tau \zeta_{d+1}}$. Furthermore, its adjoint is given by the formula

$$
\left(S_{\gamma, \tau}^{*} \varphi\right)(\alpha, \lambda)=\frac{|\lambda+\tau|^{d-|\alpha|-|\gamma|}}{|\lambda|^{d-|\alpha|}} \frac{(\alpha+\gamma) !}{\alpha !} 2^{|\gamma|} \varphi(\alpha+\gamma, \lambda+\tau)
$$

We remark that, if we call the multipliers $m_{j}, j=1, \ldots, d$, that appear in Theorem 1.3 the shift operators on $D A$, then the operators $S_{\gamma, \tau}$ correspond to the operators $\mathscr{S}^{-1} m_{j} \mathscr{S}$. Finally, we have the following result.

Theorem 1.6. Let $\left(A_{1}, \ldots, A_{d+1}\right)$ be a strongly Siegel-dissipative vector of commuting operators and set $A^{\alpha}=A_{1}^{\alpha_{1}} \cdots A_{d}^{\alpha_{d}}$ for any multi-index $\alpha \in \mathbb{N}_{0}^{d}$. Then:

(i) the map $\Theta: \mathcal{H} \rightarrow L^{2}\left(\mathbb{N}_{0}^{d} \times \mathbb{R}_{-}, d \mu ; \mathcal{H}_{\Delta}\right)$ defined as

$$
(\Theta v)(\alpha, \lambda)=\frac{|\lambda|^{|\alpha|-d}}{\alpha ! 2^{|\alpha|-\frac{1}{2}}} e^{-i \lambda A_{d+1}} A^{\alpha} v
$$

is an isometric embedding, i.e.,

$$
\|\Theta v\|_{\mathcal{L}^{2}(\Delta)}=\|v\|_{\mathcal{H}}, \quad \forall v \in \mathcal{H} .
$$

(ii) the diagram

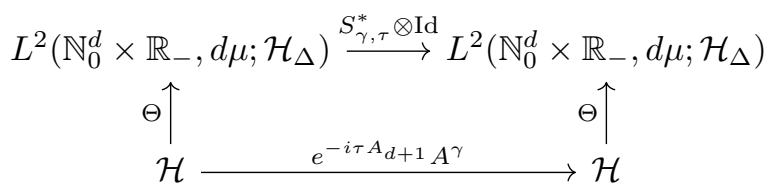

commutes. 
We conclude this introduction pointing out that the Drury-Arveson space $D A\left(\mathbb{B}^{d+1}\right)$ on the unit ball of $\mathbb{C}^{d+1}$ has drawn considerable interest since its first appearance in the works of Drury and of Arveson [7,9]. We mention $[3,5,6,10,16]$ and references therein. The Drury-Arveson space on $\mathcal{U}$ was studied in [6] and [4]; see also [8] for the case of more general Siegel type domains.

The paper is organized as follows. In Sect. 2 we recall some preliminary facts, in Sect. 3 we study the space $D A$ and we prove Theorem 1.4. In Sect. 4 we study some multiplier operators on $D A$ and we prove Theorem 1.5. We conclude proving Theorem 1.6 and Theorem 1.3 in Sect. 5.

\section{Preliminary Facts}

In this section we first recall some basic facts on the Siegel half-space, the Heisenberg group and the group Fourier transform. Then we also recall some simple properties of semigroups of operators on a Hilbert space.

\subsection{The Siegel Upper Half-space and the Heisenberg Group}

The Siegel upper half-space is defined in (1.1) and it is biholomorphically equivalent to the unit ball $\mathbb{B}^{d+1}$ via the Cayley transfom (1.2). Standard references for the facts that follow are e.g. $[11,17,20]$. We introduce new coordinates on $\mathcal{U}$ setting $\Psi\left(\zeta, \zeta_{d+1}\right)=(z, t, h)$ where

$$
\left\{\begin{array}{l}
z=\zeta \\
t=\operatorname{Re} \zeta_{d+1} \\
h=\operatorname{Im} \zeta_{d+1}-\frac{1}{4}|\zeta|^{2}
\end{array}\right.
$$

Then, if $\mathbf{U}=\mathbb{C}^{d} \times \mathbb{R} \times(0,+\infty), \Psi: \overline{\mathcal{U}} \rightarrow \overline{\mathbf{U}}$ is a $C^{\infty}$-diffeomorphism and $\Psi^{-1}$ is given by

$$
\Psi^{-1}(z, t, h)=\left(z, t+i \frac{1}{4}|z|^{2}+i h\right)=:\left(\zeta, \zeta_{d+1}\right) .
$$

Notice that $h=\varrho\left(\zeta, \zeta_{d+1}\right)$ and $\Psi\left(\zeta, t+\frac{i}{4}|\zeta|^{2}\right)=(z, t, 0)$. In this case we write $[z, t]$ in place of $(z, t, 0)$ and we also use the abuse of notation $[z, t] \in \partial \mathcal{U}$. We now let the points in $\partial \mathcal{U}$ act on $\overline{\mathcal{U}}$ as biholomorphic maps in the following way. For $[z, t] \in \partial \mathcal{U}$ set

$$
\Phi_{[z, t]}\left(\omega, \omega_{d+1}\right)=\left(\omega+z, \omega_{d+1}+t+i \frac{1}{4}|z|^{2}+\frac{i}{2} \omega \cdot \bar{z}\right),
$$

where $\omega \cdot \bar{z}=\sum_{j=1}^{d} \omega_{j} \bar{z}_{j}$ denotes the inner product in $\mathbb{C}^{d}$. Notice that

$$
\varrho\left(\Phi_{[z, t]}\left(\omega, \omega_{d+1}\right)\right)=\varrho\left(\omega, \omega_{d+1}\right)
$$


that is, the maps $\Phi_{[z, t]}$ preserve the defining function $\varrho$. In particular, for $\left(\omega, \omega_{d+1}\right) \in \partial \mathcal{U}$ and $[w, s]=\Psi\left(\omega, \omega_{d+1}\right)$, by $(2.1)$ we have

$$
\begin{aligned}
\Phi_{[z, t]}\left(\left(\omega, \omega_{d+1}\right)\right) & =\Phi_{[z, t]}\left(\Psi^{-1}(w, s, 0)\right)=\Phi_{[z, t]}\left(w, s+i \frac{1}{4}|w|^{2}\right) \\
& =\left(w+z, s+\frac{i}{4}|w|^{2}+t+\frac{i}{4}|z|^{2}+\frac{i}{2} w \cdot \bar{z}\right) \\
& =\left[w+z, s+t-\frac{1}{2} \operatorname{Im}(w \cdot \bar{z})\right] \\
& =:[w, s][z, t] .
\end{aligned}
$$

Hence, it is possible to introduce a group structure on $\partial \mathcal{U}$ itself.

Definition 2.1. The Heisenberg group $\mathbb{H}_{d}$ is the set $\mathbb{C}^{d} \times \mathbb{R}$ endowed with product

$$
[w, s][z, t]=\left[w+z, s+t-\frac{1}{2} \operatorname{Im}(w \cdot \bar{z})\right] .
$$

The right and left Haar measures on the Heisenberg group coincide with the Lebesgue measure on $\mathbb{C}^{d} \times \mathbb{R}$. In particular, the Lebesgue measure is both left and right translation invariant.

We now recall the basic facts for the Fourier transform on the Heisenberg group. For $\lambda \in \mathbb{R} \backslash\{0\}$ define the Fock space

$$
\mathcal{F}^{\lambda}=\left\{F \in \operatorname{Hol}\left(\mathbb{C}^{d}\right):\left(\frac{|\lambda|}{2 \pi}\right)^{d} \int_{\mathbb{C}^{d}}|F(z)|^{2} e^{-\frac{\lambda}{2}|z|^{2}} d z<+\infty\right\}
$$

when $\lambda>0$, and $\mathcal{F}^{\lambda}=\mathcal{F}^{|\lambda|}$ when $\lambda<0$. The Fock space is a reproducing kernel Hilbert space with reproducing kernel $e^{\frac{|\lambda|}{2} z \cdot \bar{w}}$. A complete orthonormal basis of $\mathcal{F}^{\lambda}$ is given by the normalized monomials $\left\{z^{\alpha} /\left\|z^{\alpha}\right\|_{\mathcal{F}^{\lambda}}\right\}_{\alpha \in \mathbb{N}_{0}^{d}}$, where

$$
\left\|z^{\alpha}\right\|_{\mathcal{F}^{\lambda}}^{2}=\alpha !\left(\frac{2}{|\lambda|}\right)^{|\alpha|}
$$

For $[z, t] \in \mathbb{H}_{d}$, the Bargmann representation $\sigma_{\lambda}[z, t]$ is the operator acting on $\mathcal{F}^{\lambda}$ given by,

$$
\sigma_{\lambda}[z, t] F(w)=e^{i \lambda t-\frac{\lambda}{2} w \cdot \bar{z}-\frac{\lambda}{4}|z|^{2}} F(w+z)
$$

if $\lambda>0$, and, if $\lambda<0$, as $\sigma_{\lambda}[z, t]=\sigma_{-\lambda}[\bar{z},-t]$, that is,

$$
\sigma_{\lambda}[z, t] F(w)=e^{i \lambda t+\frac{\lambda}{2} w \cdot z+\frac{\lambda}{4}|z|^{2}} F(w+\bar{z}) .
$$

If $f \in L^{1}\left(\mathbb{H}_{d}\right)$, for $\lambda \in \mathbb{R} \backslash\{0\}, \sigma_{\lambda}(f)$ is the operator acting on $\mathcal{F}^{\lambda}$ as

$$
\sigma_{\lambda}(f) F(w)=\int_{\mathbb{H}_{d}} f[z, t] \sigma_{\lambda}[z, t] F(w) d z d t .
$$

If $f \in L^{2}\left(\mathbb{H}_{d}\right)$, we have Plancherel's formula

$$
\|f\|_{L^{2}\left(\mathbb{H}_{d}\right)}^{2}=\frac{1}{(2 \pi)^{d+1}} \int_{\mathbb{R}}\left\|\sigma_{\lambda}(f)\right\|_{\mathrm{HS}}^{2}|\lambda|^{d} d \lambda,
$$

where $\left\|\sigma_{\lambda}(f)\right\|_{\mathrm{HS}}^{2}=\sum_{\alpha}\left\|\sigma_{\lambda}(f) e_{\alpha}\right\|_{\mathcal{F}^{\lambda}}^{2}$ is the Hilbert-Schmidt norm of $\sigma_{\lambda}(f)$ and $e_{\alpha}=z^{\alpha} /\left\|z^{\alpha}\right\|_{\mathcal{F} \lambda}$. If $f \in L^{1} \cap L^{2}\left(\mathbb{H}_{d}\right)$ the following inversion formula holds:

$$
f[z, t]=\frac{1}{(2 \pi)^{d+1}} \int_{\mathbb{R}} \operatorname{tr}\left(\sigma_{\lambda}(f) \sigma_{\lambda}[z, t]^{*}\right)|\lambda|^{d} d \lambda .
$$




\subsection{The Drury-Arveson Space on $\mathcal{U}$}

In [4] a family of holomorphic function spaces on $\mathcal{U}$ depending on a real parameter $\nu$ was studied and characterized by means of the group Fourier transform on $\mathbb{H}_{d}$. This family of spaces includes weighted Bergman spaces, the Hardy space, weighted Dirichlet spaces and the Dirichlet space. In particular, the Drury-Arveson space $D A$ was identified as a particular weighted Dirichlet space. Here we recall the results in [4] we need in the rest of the paper.

Definition 2.2. We define the space $\mathcal{L}_{D A}^{2}$ as the space of functions $\tau$ on $\mathbb{R} \backslash\{0\}$ such that:

$$
\begin{aligned}
& \text { (i) } \tau(\lambda): \mathcal{F}^{\lambda} \rightarrow \mathcal{F}^{\lambda} \text { is a Hilbert-Schmidt operator for every } \lambda \text {; } \\
& \text { (ii) } \tau(\lambda)=0 \text { in } \lambda>0 \\
& \text { (iii) } \operatorname{ran}(\tau(\lambda)) \subseteq \operatorname{span}\{1\} \\
& \text { (iv) }\|\tau\|_{\mathcal{L}_{D A}^{2}}^{2}:=\frac{1}{(2 \pi)^{d+1}} \int_{-\infty}^{0}\|\tau(\lambda)\|_{\mathrm{HS}}^{2}|\lambda|^{2 d} d \lambda<+\infty
\end{aligned}
$$

The following result holds true.

Theorem 2.3. ([4]) Let $n>\frac{d}{2}$. Let $f \in D A_{(n)}$ defined as in (1.3). Then, there exists $\tau \in \mathcal{L}_{D A}^{2}$ such that, for $\left(\zeta, \zeta_{d+1}\right) \in \mathcal{U}$,

$$
f\left(\zeta, \zeta_{d+1}\right)=\left(f \circ \Psi^{-1}\right)(z, t, h)=\frac{1}{(2 \pi)^{d+1}} \int_{-\infty}^{0} e^{h \lambda} \operatorname{tr}\left(\tau(\lambda) \sigma_{\lambda}[z, t]^{*}\right)|\lambda|^{d} d \lambda,
$$

and

$$
\|f\|_{D A_{(n)}}^{2}=\frac{\Gamma(2 n-d)}{2^{2 n-d}}\|\tau\|_{\mathcal{L}_{D A}^{2}}^{2} .
$$

Conversely, given $\tau \in \mathcal{L}_{D A}^{2}$, let $f$ be defined as in $(2.2)$. Then $f \in D A_{(n)}$ and $(2.3)$ holds. Therefore, for each $n>\frac{d}{2}$, the spaces $D A_{(n)}$ all coincide and their norms satisfy (2.3).

Hence, we simply write $D A$ in place of $D A_{(n)}$.

\subsection{Semigroups of Operators}

A semigroup of operators on a Hilbert space $\mathcal{H}$ is a one parameter family of bounded linear operators $\left\{T_{t}\right\}_{t \geq 0}$ on $\mathcal{H}$ such that:

(i) $T_{0}=$ Id;

(ii) $T_{t+s}=T_{t} T_{s}, \forall s, t>0$.

If in addition $T_{t}$ converges to the identity operator Id in the strong operator topology as $t \searrow 0$, the semigroup is called strongly continuous or $C_{0}$. From now on we will focus exclusively on $C_{0}$ semigroups. The infinitesimal generator $G$ of a $C_{0}$ semigroup $\left\{T_{t}\right\}_{t \geq 0}$ is a linear operator defined on the subspace $\operatorname{Dom}(G)$ of $v \in \mathcal{H}$ such that the limit

$$
\lim _{t \searrow 0} \frac{T_{t} v-v}{t}=: G v
$$

exists in the norm topology. It can be shown that $G$ on its domain $\operatorname{Dom}(G)$ is a linear densely defined closed operator [13, Section 34, Theorem 4]. In 
particular, we will be interested in the characterization of infinitesimal generators for contraction semigroups, i.e., semigroups $\left\{T_{t}\right\}_{t \geq 0}$ such that each $T_{t}$ is a contraction, which is provided by the Lumer-Philips Theorem.

Definition 2.4. A densely define operator $G$ on a Hilbert space $\mathcal{H}$ is called dissipative if

$$
\operatorname{Re}\langle G v, v\rangle_{\mathcal{H}} \leq 0, \forall v \in \operatorname{Dom}(G) .
$$

It is called maximal dissipative, if it is dissipative and its resolvent set $r(G)$ includes $\mathbb{R}_{+}=(0,+\infty)$.

For the following renowned theorem we refer the reader, for instance, to $[13$, p. 432$]$.

Theorem 2.5. (Lumer-Philips) A densely defined operator $G$ is the infinitesimal generator of a (unique) semigroup of contractions if and only if it is maximal dissipative.

The following is a very well-known lemma that we shall need in what follows.

Lemma 2.6. Let $\left\{T_{t}\right\}_{t \geq 0}$ be a semigroup of bounded operators on a Hilbert space $\mathcal{H}$. Suppose that:

(i) there exists $\delta>0$ such that $\sup _{0<t<\delta}\left\|T_{t}\right\|_{\mathcal{B}(\mathcal{H})}<+\infty$;

(ii) for some dense subset $\mathcal{D} \subseteq \mathcal{H}, T_{t} f \rightarrow f$ for all $f \in \mathcal{D}$ as $t \searrow 0 t$

Then, $\left\{T_{t}\right\}_{t \geq 0}$ is a strongly continuous semigroup.

We remark that we will work with semigroups that appear to have a negative parameter $\tau$; this is to stay consistent with [4]. When we use this notation we simply mean that the semigroup has the positive parameter $t=-\tau$.

\section{Proof of Theorem 1.4}

The proof of Theorem 1.4 follows at once from the next two lemmas. Recall that the measure $\mu$ on $\mathbb{N}_{0}^{d} \times \mathbb{R}_{-}$is defined in (1.6). Now define

$$
\varphi(\alpha, \lambda)=\left\|z^{\alpha}\right\|_{\mathcal{F}^{\lambda}}^{-1}\left\langle e_{0}, \sigma_{\lambda}\left(f_{0}\right)\left(e_{\alpha}\right)\right\rangle_{\mathcal{F}^{\lambda}} .
$$

Lemma 3.1. The map $\Phi: D A \cap H^{2} \rightarrow L^{2}\left(\mathbb{N}_{0}^{d} \times \mathbb{R}_{-}, d \mu\right)$ defined as

$$
\Phi(f)(\alpha, \lambda)=\varphi(\alpha, \lambda),
$$

extends to a conjugate linear isometry $\Phi: D A \rightarrow L^{2}\left(\mathbb{N}_{0}^{d} \times \mathbb{R}_{-}, d \mu\right)$. Its inverse is the map $\mathscr{S}$ of Theorem 1.4.

Proof. First we assume that $f \in D A \cap H^{2}$ where $H^{2}$ denotes the Hardy space on $\mathcal{U}$. This intersection is dense in $D A\left(\left[4\right.\right.$, Lemma 4.2]) and every $f \in H^{2}$ admits a boundary value function $f_{0} \in L^{2}(\partial \mathcal{U})$. Moreover, for such function $f$ the function $\tau \in \mathcal{L}_{D A}^{2}$ in formula (2.2) actually coincides with the Fourier transform of its boundary value function, that is, $\tau(\lambda)=\sigma_{\lambda}\left(f_{0}\right)$ (see $[4,14]$ ). 
Let $\left\{e_{\alpha}\right\}_{\alpha}$ be the orthonormal basis of normalized monomials of the Fock space $\mathcal{F}^{\lambda}$ and let $F=\sum_{\alpha} F_{\alpha} e_{\alpha}$ be a function in $\mathcal{F}^{\lambda}$. Then, for every function $f \in D A \cap H^{2}$, we have

$$
\sigma_{\lambda}\left(f_{0}\right)(F)=\sum_{\alpha \in \mathbb{N}_{0}^{d}} F_{\alpha} \sigma_{\lambda}\left(f_{0}\right)\left(e_{\alpha}\right)=\sum_{\alpha \in \mathbb{N}_{0}^{d}} F_{\alpha}\left\langle\sigma_{\lambda}\left(f_{0}\right)\left(e_{\alpha}\right), e_{0}\right\rangle_{\mathcal{F}^{\lambda}} e_{0}
$$

thanks to property (iii) in Definition 2.2. Thus,

$$
\sigma_{\lambda}\left(f_{0}\right)(F)=\left\langle F, \Phi_{\lambda}\right\rangle_{\mathcal{F}^{\lambda}} e_{0}
$$

where

$$
\begin{aligned}
\Phi_{\lambda} & =\sum_{\alpha \in \mathbb{N}_{0}^{d}} \overline{\left\langle\sigma_{\lambda}\left(f_{0}\right)\left(e_{\alpha}\right), e_{0}\right\rangle_{\mathcal{F}^{\lambda}}} e_{\alpha} \\
& =\sum_{\alpha \in \mathbb{N}_{0}^{d}}\left\langle e_{0}, \sigma_{\lambda}\left(f_{0}\right)\left(e_{\alpha}\right)\right\rangle_{\mathcal{F}^{\lambda}} e_{\alpha} \\
& =\sum_{\alpha \in \mathbb{N}_{0}^{d}}\left\|z^{\alpha}\right\|_{\mathcal{F}^{\lambda}}^{-1}\left\langle e_{0}, \sigma_{\lambda}\left(f_{0}\right)\left(e_{\alpha}\right)\right\rangle_{\mathcal{F}^{\lambda}} z^{\alpha} .
\end{aligned}
$$

In particular, we deduce that $\left\|z^{\alpha}\right\|_{\mathcal{F}^{\lambda}} \varphi(\alpha, \lambda)=\left\langle\Phi_{\lambda}, e_{\alpha}\right\rangle_{\mathcal{F}^{\lambda}}$. Now,

$$
\begin{aligned}
\left\|\sigma_{\lambda}\left(f_{0}\right)\right\|_{\mathrm{HS}}^{2} & =\sum_{\alpha \in \mathbb{N}_{0}^{d}}\left\|\sigma_{\lambda}\left(f_{0}\right) e_{\alpha}\right\|_{\mathcal{F}^{\lambda}}^{2} \\
& =\sum_{\alpha \in \mathbb{N}_{0}^{d}}\left|\left\langle e_{\alpha}, \Phi_{\lambda}\right\rangle_{\mathcal{F}^{\lambda}}\right|^{2}\left\|e_{0}\right\|_{\mathcal{F}^{\lambda}}^{2} \\
& =\sum_{\alpha \in \mathbb{N}_{0}^{d}}\left|\left\langle e_{\alpha}, \Phi_{\lambda}\right\rangle_{\mathcal{F}^{\lambda}}\right|^{2}
\end{aligned}
$$

since $\left\{e_{\alpha}\right\}_{\alpha}$ is an orthonormal basis in $\mathcal{F}^{\lambda}$. However,

$$
\left|\left\langle e_{\alpha}, \Phi_{\lambda}\right\rangle_{\mathcal{F}^{\lambda}}\right|=\left\|z^{\alpha}\right\|_{\mathcal{F}^{\lambda}}|\varphi(\alpha, \lambda)|,
$$

so that

$$
\|\tau(\lambda)\|_{\mathrm{HS}}^{2}=\sum_{\alpha \in \mathbb{N}_{0}^{d}} \alpha !(2 /|\lambda|)^{|\alpha|}|\varphi(\alpha, \lambda)|^{2} .
$$

In conclusion,

$$
\begin{aligned}
\|f\|_{D A}^{2} & =c_{d} \int_{-\infty}^{0} \sum_{\alpha \in \mathbb{N}_{0}^{d}} \alpha !(2 /|\lambda|)^{|\alpha|}|\varphi(\alpha, \lambda)|^{2}|\lambda|^{2 d} d \lambda \\
& =c_{d} \int_{\mathbb{N}_{0}^{d} \times \mathbb{R}_{-}}|\varphi(\alpha, \lambda)|^{2} d \mu(\alpha, \lambda) .
\end{aligned}
$$

Lemma 3.2. Let $f \in D A$. Then, with the notation above and setting $\zeta=$ $\left(\zeta_{1}, \ldots, \zeta_{d}\right)$, we have

$$
f\left(\zeta, \zeta_{d+1}\right)=\frac{1}{(2 \pi)^{d+1}} \int_{\mathbb{N}_{0}^{d} \times \mathbb{R}_{-}} \zeta^{\alpha} e^{-i \lambda \zeta_{d+1}} \overline{\varphi(\alpha, \lambda)}|\lambda|^{d} d \alpha d \lambda .
$$


Proof. If $\left(\zeta, \zeta_{d+1}\right)=\Psi^{-1}(z, t, h)$ we know from Theorem 2.3 that there exists $\tau \in \mathcal{L}_{D A}^{2}$ such that

$$
\left(f \circ \Psi^{-1}\right)(z, t, h)=\frac{1}{(2 \pi)^{d+1}} \int_{-\infty}^{0} e^{h \lambda} \operatorname{tr}\left(\tau(\lambda) \sigma_{\lambda}[z, t]^{*}\right)|\lambda|^{d} d \lambda
$$

Using the fact that $\tau(\lambda)$ is a rank one operator such that $\operatorname{rank}\{\tau(\lambda)\} \subseteq$ $\operatorname{span}\left\{e_{0}\right\}$ for every $\lambda$ we get

$$
\begin{aligned}
\operatorname{tr}\left(\tau(\lambda) \sigma_{\lambda}[z, t]^{*}\right) & =\operatorname{tr}\left(\sigma_{\lambda}[z, t]^{*} \tau(\lambda)\right) \\
& =\sum_{\alpha \in \mathbb{N}_{0}^{d}}\left\langle\tau(\lambda) e_{\alpha}, \sigma_{\lambda}[z, t] e_{\alpha}\right\rangle \\
& =\sum_{\alpha \in \mathbb{N}_{0}^{d}}\left\langle\tau(\lambda) e_{\alpha}, P_{0} \sigma_{\lambda}[z, t] e_{\alpha}\right\rangle
\end{aligned}
$$

where $P_{0}$ denotes the orthogonal projection onto the subspace generated by $e_{0}$. Moreover, it holds (see also (26) in [4])

$$
P_{0} \sigma_{\lambda}[z, t] e_{\alpha}=\left(\frac{1}{\sqrt{\alpha !}}\left(\frac{|\lambda|}{2}\right)^{|\alpha| / 2}\left(\frac{|\lambda|}{2 \pi}\right)^{d} e^{i \lambda t+\frac{\lambda}{4}|z|^{2}} \bar{z}^{\alpha}\right) e_{0}
$$

Thus,

$$
\begin{aligned}
\operatorname{tr}\left(\tau(\lambda) \sigma_{\lambda}[z, t]^{*}\right) & =\sum_{\alpha \in \mathbb{N}_{0}^{d}}\left\langle\tau(\lambda) e_{\alpha}, P_{0} \sigma_{\lambda}[z, t] e_{\alpha}\right\rangle \\
& =\sum_{\alpha \in \mathbb{N}_{0}^{d}}\left\langle e_{\alpha}, \Phi_{\lambda}\right\rangle\left\langle e_{0}, P_{0} \sigma_{\lambda}[z, t] e_{\alpha}\right\rangle \\
& =\sum_{\alpha \in \mathbb{N}_{0}^{d}}\left\langle e_{\alpha}, \Phi_{\lambda}\right\rangle \overline{\left\langle P_{0} \sigma_{\lambda}[z, t] e_{\alpha}, e_{0}\right\rangle} \\
& =\sum_{\alpha \in \mathbb{N}_{0}^{d}} \frac{1}{\sqrt{\alpha !}}\left(\frac{|\lambda|}{2}\right)^{|\alpha| / 2} e^{-i \lambda t+\frac{\lambda}{4}|z|^{2}} z^{\alpha}\left\langle e_{\alpha}, \Phi_{\lambda}\right\rangle \\
& =\sum_{\alpha \in \mathbb{N}_{0}^{d}} \frac{1}{\sqrt{\alpha !}}\left(\frac{|\lambda|}{2}\right)^{|\alpha| / 2}\left\|z^{\alpha}\right\| \overline{\varphi(\alpha, \lambda)} e^{-i \lambda t+\frac{\lambda}{4}|z|^{2}} z^{\alpha} . \\
& =\sum_{\alpha \in \mathbb{N}_{0}^{d}} \frac{\varphi(\alpha, \lambda)}{\varphi} e^{-i \lambda t+\frac{\lambda}{4}|z|^{2}} z^{\alpha},
\end{aligned}
$$


where the series converges absolutely since $\phi \in L^{2}\left(\mathbb{N}_{0}^{d} \times \mathbb{R}_{-}, d \mu\right)$. Hence by $(2.2)$,

$$
\begin{aligned}
f\left(\zeta, \zeta_{d+1}\right) & =\left(f \circ \Psi^{-1}\right)(z, t, h) \\
& =\frac{1}{(2 \pi)^{d+1}} \int_{-\infty}^{0} e^{h \lambda} \sum_{\alpha \in \mathbb{N}_{0}^{d}} \overline{\varphi(\alpha, \lambda)} e^{-i \lambda t+\frac{\lambda}{4}|z|^{2}} z^{\alpha}|\lambda|^{d} d \lambda \\
& =\frac{1}{(2 \pi)^{d+1}} \int_{\mathbb{N}_{0}^{d} \times \mathbb{R}_{-}} z^{\alpha} e^{-i \lambda\left(t-i\left(\frac{|z|^{2}}{4}+h\right)\right)} \overline{\varphi(\alpha, \lambda)}|\lambda|^{d} d \alpha d \lambda \\
& =\frac{1}{(2 \pi)^{d+1}} \int_{\mathbb{N}_{0}^{d} \times \mathbb{R}_{-}} \zeta^{\alpha} e^{-i \lambda \zeta_{d+1}} \overline{\varphi(\alpha, \lambda)}|\lambda|^{d} d \alpha d \lambda
\end{aligned}
$$

as we wished to show.

\section{Pointwise Multipliers on the Drury-Arveson Space}

In this section we explicitly study some multiplier operators on $D A$ and we prove Theorem 1.5. Recall that given a function $m$ the associated multiplier operator is the operator $f \mapsto m f$. The problem of characterizing the multiplier algebra of a given reproducing kernel Hilbert function space is a classical problem. In the case of the Drury-Arveson space on the unit ball $D A\left(\mathbb{B}^{d+1}\right)$ this problem turned out to be very challenging; a first important result is due to J. Ortega and J. Fäbrega [15]. Their result reads as follows. Let $n \in \mathbb{N}_{0}$ be such that $2 n>d$ and for $f$ in $\operatorname{Hol}\left(\mathbb{B}^{d+1}\right)$ define the measure

$$
d \nu_{f}(w)=\left|\mathcal{R}^{n} f(w)\right|^{2}\left(1-|w|^{2}\right)^{2 n-d-1} d \nu(w),
$$

where $\mathcal{R}$ is the radial derivative, $d \nu$ is the normalized Lebesgue measure on $\mathbb{B}^{d+1}$ and $w \in \mathbb{B}^{d+1}$. Then, $f$ is a $D A\left(\mathbb{B}^{d+1}\right)$-multiplier if and only if $f \in H^{\infty}\left(\mathbb{B}^{d+1}\right)$ and $d \nu_{f}$ is a Carleson measure for $D A\left(\mathbb{B}^{d+1}\right)$. A few years later N. Arcozzi, R. Rochberg and E. Sawyer in [5] completely characterized the Carleson measures of $D A\left(\mathbb{B}^{d+1}\right)$; see also $[18,19,21]$. Hence, the multiplier algebra of the Drury-Arveson space on the unit ball is completely characterized. Nonetheless, there is still interest in finding an easier characterization and we refer the reader, for instance, to [10].

From $[5,15]$ we can also deduce an indirect characterization of the multiplier algebra for $D A$ on the Siegel half-space. Indeed, let $\mathcal{C}$ be the multidimensional Cayley transform defined in (1.2). Then, up to an irrelevant multiplicative constant,

$$
\begin{aligned}
K_{D A}^{\mathcal{U}}\left(\mathcal{C}\left(z, z_{d+1}\right), \mathcal{C}\right. & \left.\left(w, w_{d+1}\right)\right) \\
= & \left(1-z_{d+1}\right) K_{D A}^{\mathbb{B}^{d+1}}\left(\left(z, z_{d+1}\right),\left(w, w_{d+1}\right)\right)\left(1-\overline{w_{d+1}}\right)
\end{aligned}
$$

where $K_{D A}^{\mathcal{U}}$ and $K_{D A}^{\mathbb{B}^{d+1}}$ denote the reproducing kernel of $D A$ and $D A\left(\mathbb{B}^{d+1}\right)$ respectively, and $\left(z, z_{d+1}\right),\left(w, w_{d+1}\right) \in \mathbb{B}^{d+1}$. From the abstract theory of reproducing kernel Hilbert spaces (see, for instance, [1, Chapter 2.6]) we deduce that

$$
f \mapsto\left(1-z_{d+1}\right)^{-1}(f \circ \mathcal{C})
$$


is a surjective isometry from $D A$ onto $D A\left(\mathbb{B}^{d+1}\right)$ and that $m$ is a multiplier for $D A$ if and only if $(m \circ \mathcal{C})$ is a multiplier for $D A\left(\mathbb{B}^{d+1}\right)$.

For our goal we need to study the multiplier operators associated to the functions $\zeta^{\gamma}=\zeta_{1}^{\gamma_{1}} \cdots \zeta_{d}^{\gamma_{d}}, e^{-i \tau \zeta_{d+1}}$ for $\tau<0$ and $\zeta^{\gamma} e^{-i \tau \zeta_{d+1}}$. However, we do not rely on the multiplier characterization on the unit ball since it is easier to study them directly. The proof of the following lemma is standard and we omit it.

Lemma 4.1. The set

$\mathcal{D}=\left\{f \in L^{2}\left(\mathbb{N}_{0}^{d} \times \mathbb{R}_{-}, d \mu\right): \operatorname{supp} f\right.$ is compact and $\left.f(\alpha, \cdot) \in C_{c}^{\infty} \forall \alpha \in \mathbb{N}_{0}^{d}\right\}$. is dense in $L^{2}\left(\mathbb{N}_{0}^{d} \times \mathbb{R}_{-}, d \mu\right)$.

We now study the multiplier operator associated to the monomial $\zeta^{\gamma}, \gamma \in$ $\mathbb{N}_{0}^{d}$. Although this operator is unbounded on $D A$, as it is easily seen, it is closed and densely defined.

Lemma 4.2. Let $\gamma \in \mathbb{N}_{0}^{d}$. Then, the multiplier operator $m_{\gamma}$ associated to the function $\zeta^{\gamma}=\zeta_{1}^{\gamma_{1}} \cdots \zeta_{d}^{\gamma_{d}}$ is a closed densely defined operator on $D A$.

Proof. Let us consider $\mathcal{D}_{\gamma}=\left\{f \in D A: \zeta^{\gamma} f \in D A\right\}$ as domain of our multiplier operator. Let $\left\{f_{n}\right\}_{n} \subseteq \mathcal{D}_{\gamma}$ be a sequence such that $f_{n} \rightarrow f$ in $D A$ and $\zeta^{\gamma} f_{n} \rightarrow g \in D A$. Since $D A$ is a reproducing kernel Hilbert space we also have that $f_{n} \rightarrow f$ and $\zeta^{\gamma} f_{n} \rightarrow g$ uniformly on compact sets. Hence, $\zeta^{\gamma} f_{n} \rightarrow \zeta^{\gamma} f$ uniformly on compact sets as well and $\zeta^{\gamma} f=g$. In particular both $f$ and $\zeta^{\gamma} f$ are in $D A$ so that $f$ belongs to the domain $\mathcal{D}_{\gamma}$. Thus, our operator is closed.

To prove that $\mathcal{D}_{\gamma}$ is dense we exploit the previous Lemma 4.1. Let $\varphi \in \mathcal{D}$ and define $f \in D A$ by (3.3). Then,

$$
\begin{aligned}
\zeta^{\gamma} f\left(\zeta, \zeta_{d+1}\right) & =\frac{1}{(2 \pi i)^{d+1}} \int_{\mathbb{N}_{0}^{d} \times \mathbb{R}_{-}} \zeta^{\alpha+\gamma} e^{-i \lambda \zeta_{d+1}} \overline{\varphi(\alpha, \lambda)}|\lambda|^{d} d \alpha d \lambda \\
& =\frac{1}{(2 \pi)^{d+1}} \int_{\operatorname{supp} \varphi} \zeta^{\alpha} e^{-i \lambda \zeta_{d+1}} \overline{\varphi(\alpha-\gamma, \lambda)}|\lambda|^{d} \chi_{\{\alpha \geq \gamma\}}(\alpha) d \alpha d \lambda
\end{aligned}
$$

where $\chi_{\{\alpha \geq \gamma\}}(\alpha)$ is the characteristic function of the set $\left\{\alpha \in \mathbb{N}_{0}^{d}: \alpha_{i} \geq\right.$ $\left.\gamma_{i}, i=1, \ldots, d\right\}$. Recall that $\operatorname{supp} \varphi$ is a compact subset of $\mathbb{N}_{0}^{d} \times \mathbb{R}_{-}$and assume that $(\alpha, \lambda) \in \operatorname{supp} \varphi$ implies $|\alpha|<N$ for some positive integer $N$ and $\lambda \in I$ where $I$ is a compact subset of $\mathbb{R}_{-}$. From (3.2) we have

$$
\begin{aligned}
\left\|\zeta^{\gamma} f\right\|_{D A}^{2} & =c_{d} \int_{I} \sum_{|\alpha-\gamma|<N}|\varphi(\alpha-\gamma, \lambda)|^{2} \alpha !\left(\frac{2}{|\lambda|}\right)^{|\alpha|}|\lambda|^{2 d} \chi_{\{\alpha \geq \gamma\}}(\alpha) d \alpha d \lambda \\
& =c_{d} \int_{I} \sum_{|\alpha|<N}|\varphi(\alpha, \lambda)|^{2} \frac{(\alpha+\gamma) !}{\alpha !}\left(\frac{2}{|\lambda|}\right)^{|\gamma|} d \mu(\alpha, \lambda) \\
& \leq c_{d}(\varphi) \int_{I} \sum_{|\alpha|<N}|\varphi(\alpha, \lambda)|^{2} d \mu(\alpha, \lambda) \\
& =c_{d}(\varphi)\|f\|_{D A}^{2}
\end{aligned}
$$


where $c_{d}(\varphi)$ is a constant depending on the compact support of $\varphi$ (and on the dimension $d$ ). The density of $\mathcal{D}_{\gamma}$ in $D A$ now follows from the density of $\mathcal{D}$ in $L^{2}\left(\mathbb{N}_{0}^{d} \times \mathbb{R}_{-}, d \mu\right)$ and Theorem 1.4.

We now investigate the other multiplier operators we are interested in and see that they are actually bounded on the Drury-Arveson space.

Lemma 4.3. The pointiwise multiplier operators associated to the functions $\left\{e^{-i \tau \zeta_{d+1}}\right\}_{\tau<0}$ form a strongly continuous semigroup of contractions on DA with corresponding infinitesimal generator the multiplier operator corresponding to the function $i \zeta_{d+1}$.

Proof. From the inversion formula (3.3) we have

$$
\begin{aligned}
e^{-i \tau \zeta_{d+1}} f\left(\zeta, \zeta_{d+1}\right) & =\frac{1}{(2 \pi i)^{d+1}} \int_{\mathbb{N}_{0}^{d} \times \mathbb{R}_{-}} \zeta^{\alpha} e^{-i(\lambda+\tau) \zeta_{d+1}} \overline{\varphi(\alpha, \lambda)}|\lambda|^{d} d \alpha d \lambda \\
& =\frac{1}{(2 \pi i)^{d+1}} \int_{\mathbb{N}_{0}^{d} \times \mathbb{R}_{-}} \zeta^{\alpha} e^{-i \lambda \zeta_{d+1}} \overline{\varphi(\alpha, \lambda-\tau)}\left|\frac{\lambda-\tau}{\lambda}\right|^{d} \chi_{(-\infty, \tau)}(\lambda)|\lambda|^{d} d \alpha d \lambda
\end{aligned}
$$

where $\chi_{(-\infty, \tau)}$ is the characteristic function of the interval $(-\infty, \tau)$. Thus, by (3.2) we get

$$
\begin{aligned}
\| e^{-i \tau \zeta_{d+1} f \|_{D A}^{2}} & =\int_{\mathbb{N}_{0}^{d} \times \mathbb{R}_{-}}|\varphi(\alpha, \lambda-\tau)|^{2}\left|\frac{\lambda-\tau}{\lambda}\right|^{2 d} \chi_{(-\infty, \tau)}(\lambda) d \mu(\alpha, \lambda) \\
& =\int_{\mathbb{N}_{0}^{d} \times \mathbb{R}_{-}}|\varphi(\alpha, \lambda)|^{2}\left|\frac{\lambda}{\lambda+\tau}\right|^{|\alpha|} d \mu(\alpha, \lambda) \\
& \leq \int_{\mathbb{N}_{0}^{d} \times \mathbb{R}_{-}}|\varphi(\alpha, \lambda)|^{2} d \mu(\alpha, \lambda) \\
& =\|f\|_{D A}^{2} .
\end{aligned}
$$

Hence, we conclude that $e^{-i \tau \zeta_{d+1}}$ is a contractive multiplier. The semigroup property is automatically satisfied. Since $e^{-i \tau \zeta_{d+1}} f$ converges in norm to $f$ for all $f \in \mathcal{D}$ and the semigroup has uniformly bounded seminorm, it is strongly continuous by Lemma 2.6. Then, by definition, the infinitesimal generator is given by

$$
\lim _{\tau \rightarrow 0^{-}} \frac{\left(e^{-i \tau \zeta_{d+1}}-1\right) f}{-\tau}=i \zeta_{d+1} f
$$

and the proof is concluded.

Lemma 4.4. Let $(\gamma, \tau) \in \mathbb{N}_{0}^{d} \times \mathbb{R}_{-}, \tau<0$. Then, the multiplier operator associated to the muliplier $\zeta^{\gamma} e^{-i \tau \zeta_{d+1}}$ extends to a bounded operator on DA.

Proof. Let $\mathcal{D}$ be as in Lemma 4.1 and let $f \in \mathcal{D}$. We have

$$
\begin{aligned}
& \zeta^{\gamma} e^{-i \tau \zeta_{d+1}} f\left(\zeta, \zeta_{d+1}\right)=\frac{1}{(2 \pi i)^{d+1}} \int_{\mathbb{N}_{0}^{d} \times \mathbb{R}_{-}} \zeta^{\alpha+\gamma} e^{-i(\lambda+\tau) \zeta_{d+1}} \overline{\varphi(\alpha, \lambda)}|\lambda|^{d} d \alpha d \lambda \\
& =\frac{1}{(2 \pi i)^{d+1}} \int_{\mathbb{N}_{0}^{d} \times \mathbb{R}_{-}} \zeta^{\alpha} e^{-i \lambda \zeta_{d+1}} \overline{\varphi(\alpha-\gamma, \lambda-\tau)}|\lambda-\tau|^{d} \chi_{(-\infty, \tau)}(\lambda) \chi_{\{\alpha \geq \gamma\}}(\alpha) d \alpha d \lambda \\
& =\frac{1}{(2 \pi i)^{d+1}} \int_{\mathbb{N}_{0}^{d} \times \mathbb{R}_{-}} \zeta^{\alpha} e^{-i \lambda \zeta_{d+1}} \overline{\varphi(\alpha-\gamma, \lambda-\tau)}\left|\frac{\lambda-\tau}{\lambda}\right|^{d} \chi_{(-\infty, \tau)}(\lambda) \chi_{\{\alpha \geq \gamma\}}(\alpha)|\lambda|^{d} d \alpha d \lambda
\end{aligned}
$$


where $\chi_{\{\alpha \geq \gamma\}}(\alpha)$ is the characteristic function of the set $\left\{\alpha \in \mathbb{N}_{0}^{d}: \alpha_{i} \geq\right.$ $\left.\gamma_{i}, i=1, \ldots, d\right\}$. Hence, setting $m_{\gamma, \tau}\left(\zeta, \zeta_{d+1}\right)=\zeta^{\gamma} e^{-i \tau \zeta_{d+1}}$,

$$
\begin{aligned}
\left\|m_{\gamma, \tau} f\right\|_{D A}^{2} & \\
& =\int_{\mathbb{N}_{0}^{d} \times \mathbb{R}_{-}}|\varphi(\alpha-\gamma, \lambda-\tau)|^{2}\left|1-\frac{\tau}{\lambda}\right|^{2 d} \chi_{(-\infty, \tau)}(\lambda) \chi_{\{\alpha \geq \gamma\}}(\alpha) d \mu(\alpha, \lambda) \\
& =\int_{\mathbb{N}_{0}^{d} \times \mathbb{R}_{-}}|\varphi(\alpha, \lambda)|^{2} 2^{|\gamma|} \frac{|\lambda|^{|\alpha|}}{|\lambda+\tau|^{|\alpha+\gamma|}} \frac{(\alpha+\gamma) !}{\alpha !} d \mu(\alpha, \lambda) .
\end{aligned}
$$

Therefore,

$$
\left\|m_{\gamma, \tau}\right\|_{\mathcal{M}(D A)}^{2} \leq \sup _{\alpha \in \mathbb{N}_{0}^{d}, \lambda<0} 2^{|\gamma|} \frac{|\lambda|^{|\alpha|}}{|\lambda+\tau|^{|\alpha+\gamma|}} \frac{(\alpha+\gamma) !}{\alpha !} .
$$

In order to calculate this supremum we first maximize over $\lambda$. For symmetry we can consider $\lambda>0$ and $\tau>0$. We find that when $|\alpha| \neq 0$ the maximum is achieved for $\lambda=\frac{|\alpha|}{|\gamma|} \tau$, whereas for $\alpha=0$ the expression is decreasing in $\lambda$ and therefore the maximum is achieved at $\lambda=0$.

Substituting the value for $\lambda$ in the expression above we find

$$
\begin{aligned}
& 2^{|\gamma|} \frac{|\alpha|^{|\alpha|} \tau^{|\alpha|}}{|\gamma|^{|\alpha|}} \frac{1}{\tau^{|\alpha+\gamma|}\left(\frac{|\alpha|}{|\gamma|}+1\right)^{|\alpha+\gamma|}} \frac{(\alpha+\gamma) !}{\alpha !}= \\
& \quad=\left(\frac{2}{\tau}\right)^{|\gamma|}\left(1+\frac{|\gamma|}{|\alpha|}\right)^{-|\alpha|} \frac{(\alpha+\gamma) !}{\alpha !}\left(\frac{|\alpha|}{|\gamma|}+1\right)^{-|\gamma|} .
\end{aligned}
$$

Note that the first factor is constant, the second is decreasing in $|\alpha|$ and tends to $e^{-|\gamma|}$ as $|\alpha| \rightarrow+\infty$. For the last term notice that

$$
\begin{aligned}
\frac{(\alpha+\gamma) !}{\alpha !}\left(\frac{|\alpha|}{|\gamma|}+1\right)^{-|\gamma|} & =\prod_{i=1}^{d} \frac{\left(\alpha_{i}+\gamma_{i}\right) !}{\alpha_{i} !\left(\frac{|\alpha|}{|\gamma|}+1\right)^{\gamma_{i}}} \\
& \leq \prod_{i=1}^{d} \frac{(|\alpha|+\gamma) !}{|\alpha| !\left(\frac{|\alpha|}{|\gamma|}+1\right)^{\gamma_{i}}} \\
& \leq \gamma ! \prod_{i=1}^{d}\left(\frac{|\alpha|+1}{\frac{|\alpha|}{|\gamma|}+1}\right)^{\gamma_{i}} \\
& \leq \gamma !|\gamma|^{|\gamma|}
\end{aligned}
$$

This proves that the multiplier operator associated to $m_{\gamma, \alpha}$ is bounded with norm less than

$$
\max \left\{\gamma !\left(\frac{2}{\tau}\right)^{|\gamma|}, \gamma !\left(\frac{2}{\tau}\right)^{|\gamma|} \frac{|\gamma| !}{\sqrt{2 \pi|\gamma|}}\right\}
$$

where we used Stirling's asymptotic.

We now prove Theorem 1.5 
Proof. The proof of the unitary equivalence of the two operators follows essentially from the computation in Lemma 4.4. For the computation of the adjoint operator, if $\langle\cdot, \cdot\rangle$ denotes the inner product in $L^{2}\left(\mathbb{N}_{0}^{d} \times \mathbb{R}_{-}, d \mu\right)$, we have

$$
\begin{aligned}
\left\langle S_{\gamma, \tau}^{*} \varphi, \psi\right\rangle & =\left\langle\varphi, S_{\gamma, \tau} \psi\right\rangle \\
& =\int_{\{\alpha \geq \gamma\}} \int_{\{\lambda<\tau\}} \varphi(\alpha, \lambda) \frac{|\lambda-\tau|^{d}}{|\lambda|^{d}} \overline{\psi(\alpha-\gamma, \lambda-\tau)} d \mu(\alpha, \lambda) \\
& =\int_{\mathbb{N}_{0}^{d} \times \mathbb{R}_{-}} \frac{|\lambda+\tau|^{d-|\alpha|-|\gamma|}}{|\lambda|^{d-|\alpha|}} \frac{(\alpha+\gamma) !}{\alpha !} 2^{|\gamma|} \varphi(\alpha+\gamma, \lambda+\tau) \overline{\psi(\alpha, \lambda)} d \mu(\alpha, \lambda)
\end{aligned}
$$

and the conclusion follows.

\section{The von Neumann Type Inequality}

In this section we prove our main results Theorem 1.6 and Theorem 1.3. We first prove a couple of lemmas.

Lemma 5.1. Let $T$ be a bounded operator on a Hilbert space $\mathcal{H}$ and let $U$ be a densely defined closed operator. Assume that $U$ is the infinitesimal generator of a (unique) $C_{0}$ semigroup $\left\{e^{\tau U}\right\}_{\tau>0}$ and assume that $T$ and $U$ strongly commute. Then, $T$ and $e^{\tau U}$ commute for all $\tau>0$.

Proof. Consider some $\lambda_{0} \in r(T)$, the resolvent set of the operator $T$. Then an algebraic computation shows that the bounded operators

$$
Z_{\tau}:=\left(T-\lambda_{0} \mathrm{Id}\right)^{-1} e^{\tau U}\left(T-\lambda_{0} \mathrm{Id}\right)
$$

form a $C_{0}$-semigroup. For $v \in \operatorname{Dom}(U)$, since $T$ and $U$ strongly commute, $T h-\lambda_{0} v \in \operatorname{Dom}(U)$ and

$$
\begin{aligned}
\lim _{\tau \searrow 0} \frac{Z_{\tau} v-v}{\tau} & =\left(T-\lambda_{0} \mathrm{Id}\right)^{-1} \lim _{\tau \searrow 0} \frac{e^{\tau U}\left(T-\lambda_{0} \mathrm{Id}\right) v-\left(T-\lambda_{0} \mathrm{Id}\right) v}{\tau} \\
& =\left(T-\lambda_{0} \mathrm{Id}\right)^{-1} U\left(T v-\lambda_{0} v\right)=U v
\end{aligned}
$$

By the uniqueness of the infinitesimal generator we conclude that $Z_{\tau}=e^{\tau U}$. In other words,

$$
e^{\tau U} T=T e^{\tau U}
$$

for all $\tau>0$, as we wished to show.

Proof of Theorem 1.6. Set $A=\left(A_{1} \cdots A_{d}\right)$, let $v \in \mathcal{H}$ and notice that, since for $1 \leq i \leq d$ the operators $A_{i}$ strongly commute with $A_{d+1}$, by [13, Section 34, Theorem 4(i)] we can infer that $e^{-i \tau A_{d+1}} A^{\alpha} v \in \operatorname{Dom} A_{d+1}$. Therefore, 
the map $\Theta$ is well-defined. Furthermore,

$$
\begin{aligned}
\|\Theta v\|_{\mathcal{L}^{2}(\Delta)}^{2}= & \int_{\mathbb{N}_{0}^{d} \times \mathbb{R}_{-}}\|\Theta v(\alpha, \lambda)\|_{\Delta}^{2} d \mu(\alpha, \lambda) \\
= & \int_{\mathbb{N}_{0}^{d} \times \mathbb{R}_{-}}\left\|e^{-i \lambda A_{d+1}} A^{\alpha} v\right\|_{\Delta}^{2} \frac{|\lambda|^{|\alpha|}}{2^{|\alpha|-1} \alpha !} d \alpha d \lambda \\
= & \int_{\mathbb{N}_{0}^{d} \times \mathbb{R}_{-}} \operatorname{Im}\left\langle A_{d+1} e^{-i \lambda A_{d+1}} A^{\alpha} v, e^{-i \lambda A_{d+1}} A^{\alpha} v\right\rangle_{\mathcal{H}} \frac{|\lambda|^{|\alpha|}}{2^{|\alpha|-1} \alpha !} d \alpha d \lambda \\
& -\int_{\mathbb{N}_{0}^{d} \times \mathbb{R}_{-}} \sum_{i=1}^{d}\left\langle A^{\alpha+e_{i}} e^{-i \lambda A_{d+1}} v, A^{\alpha+e_{i}} e^{-i \lambda A_{d+1}} v\right\rangle_{\mathcal{H}} \frac{|\lambda|^{|\alpha|}}{2^{|\alpha|+1} \alpha !} d \alpha d \lambda .
\end{aligned}
$$

Now we observe that for $v \in \operatorname{Dom} A_{d+1}$ we have

$$
\begin{aligned}
\partial_{\lambda}\left\|e^{-i \lambda A_{d+1}} v\right\|_{\mathcal{H}}^{2}=\langle & \left.-i A_{d+1} e^{-i \lambda A_{d+1}} v, e^{-i \lambda A_{d+1}} v\right\rangle_{\mathcal{H}} \\
& +\left\langle e^{-i \lambda A_{d+1}} v,-i A_{d+1} e^{-i \lambda A_{d+1}} v\right\rangle_{\mathcal{H}} \\
= & 2 \operatorname{Im}\left\langle A_{d+1} e^{-i \lambda A_{d+1}} v, e^{-i \lambda A_{d+1}} v\right\rangle_{\mathcal{H}} .
\end{aligned}
$$

From the fact that $\left(A_{1}, \ldots, A_{d+1}\right)$ is strongly Siegel-dissipative we deduce that $i A_{d+1}+\varepsilon \mathrm{Id}$ is maximal dissipative. In this case the Lumer-Philips Theorem guarantees that $i A_{d+1}+\varepsilon$ Id generates a contraction semigroup; in other words, for any $v \in \mathcal{H}, \tau<0$,

$$
e^{-\tau \varepsilon}\left\|e^{-i \tau A_{d+1}} v\right\|_{\mathcal{H}} \leq\|v\|_{\mathcal{H}} .
$$

Therefore, $\left\|e^{-i \tau A_{d+1}} v\right\|_{\mathcal{H}}$ decays exponentially and we can integrate by parts as follows,

$$
\begin{aligned}
& \|\Theta v\|_{\mathcal{L}^{2}(\Delta)}^{2}=\sum_{\alpha \in \mathbb{N}_{0}^{d}} \int_{-\infty}^{0} \partial_{\lambda}\left\|e^{-i \lambda A_{d+1}} A^{\alpha} v\right\|_{\mathcal{H}}^{2} \frac{|\lambda|^{|\alpha|}}{2^{|\alpha|} \alpha !} d \lambda \\
& -\sum_{\alpha \in \mathbb{N}_{0}^{d}} \int_{-\infty}^{0} \sum_{i=1}^{d}\left\|A^{\alpha+e_{i}} e^{-i \lambda A_{d+1}} v\right\|_{\mathcal{H}}^{2} \frac{|\lambda|^{|\alpha|}}{2^{|\alpha|+1} \alpha !} d \lambda \\
& =\|v\|_{\mathcal{H}}^{2}+\sum_{\alpha \in \mathbb{N}_{0}^{d} \backslash\{0\}} \int_{-\infty}^{0} \partial_{\lambda}\left\|e^{-i \lambda A_{d+1}} 2^{-\frac{|\alpha|}{2}} A^{\alpha} v\right\|_{\mathcal{H}}^{2} \frac{|\lambda|^{|\alpha|}}{\alpha !} d \lambda \\
& -\sum_{i=1}^{d} \sum_{\alpha \in \mathbb{N}_{0}^{d}} \int_{-\infty}^{0}\left\|e^{-i \lambda A_{d+1}} 2^{-\frac{|\alpha|+1}{2}} A^{\alpha+e_{i}} v\right\|_{\mathcal{H}}^{2} \frac{|\lambda|^{|\alpha|}}{\alpha !} d \lambda \\
& =\|v\|_{\mathcal{H}}^{2}+\sum_{\alpha \in \mathbb{N}_{0}^{d} \backslash\{0\}} \int_{-\infty}^{0}\left\|e^{-i \lambda A_{d+1}} 2^{-\frac{|\alpha|}{2}} A^{\alpha} v\right\|_{\mathcal{H}}^{2} \frac{|\alpha \| \lambda|^{|\alpha|-1}}{\alpha !} d \lambda \\
& -\sum_{\alpha \in \mathbb{N}_{0}^{d}} \int_{-\infty}^{0} \sum_{i=1}^{d}\left\|e^{-i \lambda A_{d+1}} 2^{-\frac{|\alpha|+1}{2}} A^{\alpha+e_{i}} v\right\|_{\mathcal{H}}^{2} \frac{|\lambda|^{|\alpha|}}{\alpha !} d \lambda \\
& =\|v\|_{\mathcal{H}}^{2} .
\end{aligned}
$$


To see why the two sums cancel each other out, notice that

$$
\begin{aligned}
\left\|e^{-i \lambda A_{d+1}} 2^{-\frac{|\alpha|+1}{2}} A^{\alpha+e_{i}} v\right\|_{\mathcal{H}}^{2} & \frac{|\lambda|^{|\alpha|}}{\alpha !} \\
& =\left\|e^{-i \lambda A_{d+1}} 2^{-\frac{\left|\alpha+e_{i}\right|}{2}} A^{\alpha+e_{i}} v\right\|_{\mathcal{H}}^{2} \frac{\left(\alpha_{i}+1\right)|\lambda|^{\left|\alpha+e_{i}\right|-1}}{\left(\alpha+e_{i}\right) !}
\end{aligned}
$$

and use the combinatorial identity

$$
\sum_{i=1}^{d} \sum_{\alpha \in \mathbb{N}_{0}^{d}} c_{\alpha+e_{i}}\left(\alpha_{i}+1\right)=\sum_{i=1}^{d} \sum_{\beta: \beta_{i} \geq 1} c_{\beta} \beta_{i}=\sum_{\beta \in \mathbb{N}_{0}^{d} \backslash\{0\}} c_{\beta} \sum_{i: \beta_{i} \geq 1} \beta_{i}=\sum_{\beta \in \mathbb{N}_{0}^{d} \backslash\{0\}} c_{\beta}|\beta| .
$$

We now lift the operator $S_{\gamma, \tau}^{*}$ to the space $\mathcal{L}^{2}(\Delta)$ by tensoring with the identity. Explicitly, for $g: \mathbb{N}_{0}^{d} \rightarrow \mathcal{H}_{\Delta}$,

$$
\left[S_{\gamma, \tau}^{*} \otimes \operatorname{Id}\right] g(\alpha, \tau)=\frac{|\lambda+\tau|^{d-|\alpha|-|\gamma|}}{|\lambda|^{d-|\alpha|}} \frac{(\alpha+\gamma) !}{\alpha !} 2^{|\gamma|} g(\alpha+\gamma, \lambda+\tau) .
$$

We now show that the operator $\Theta$ is an intertwining operator for the couple $S_{\gamma, \tau}^{*} \otimes \operatorname{Id}$ and $e^{-i \tau A_{d+1}} A^{\gamma}$ where $A=\left(A_{1}, \ldots, A_{d}\right)$. For, we have

$$
\Theta\left(e^{-i \tau A_{d+1}} A^{\gamma} v\right)(\alpha, \lambda)=\frac{|\lambda|^{|\alpha|-d}}{\alpha ! 2^{|\alpha|-\frac{1}{2}}} e^{-i(\tau+\lambda) A_{d+1}} A^{\alpha+\gamma} v,
$$

whereas, on the other hand,

$$
\begin{aligned}
\left(\left[S_{\gamma, \tau}^{*}\right.\right. & \otimes \operatorname{Id}] \Theta v)(\alpha, \lambda)=\frac{|\lambda+\tau|^{d-|\alpha|-|\gamma|}}{|\lambda|^{d-|\alpha|}} \frac{(\alpha+\gamma) !}{\alpha !} 2^{|\gamma|} \Theta v(\alpha+\gamma, \lambda+\tau) \\
& =\frac{|\lambda+\tau|^{d-|\alpha|-|\gamma|}}{|\lambda|^{d-|\alpha|}} \frac{(\alpha+\gamma) !}{\alpha !} 2^{|\gamma|} \frac{|\lambda+\tau|^{|\alpha|+|\gamma|-d}}{(\alpha+\gamma) ! 2^{|\alpha|+|\gamma|-\frac{1}{2}}} e^{-i(\tau+\lambda) A_{d+1} A^{\alpha+\gamma} v} \\
& =\frac{|\lambda|^{|\alpha|-d}}{\alpha ! 2^{|\alpha|-\frac{1}{2}}} e^{-i(\tau+\lambda) A_{d+1}} A^{\alpha+\gamma} v \\
& =\Theta\left(e^{-i \tau A_{d+1}} A^{\gamma} v\right)(\alpha, \lambda) .
\end{aligned}
$$

In conclusion, the diagram (1.7) commutes and (ii) is proved.

Finally, we prove our von Neumann type inequality.

Proof of Theorem 1.3. First suppose that the tuple $\left(A_{1}, \ldots A_{d+1}\right)$ is strongly Siegel-dissipative. Let $p(z)=\sum_{|\gamma| \leq N} c_{\gamma} z^{\gamma}$ be a polynomial in $z=\left(z_{1}, \ldots, z_{d}\right)$. Let $\left(\tau_{1}, \ldots, \tau_{d}\right) \in \mathbb{R}_{-}^{d}$ be fixed and let $M_{j}$ be the operator on $\mathcal{H}$ given by $M_{j}=e^{-i \tau_{j} A_{d+1}} A_{j}, j=1, \ldots, d$. Then,

$$
p\left(M_{1}, \ldots, M_{d}\right)=\sum_{|\gamma| \leq N} c_{\gamma} e^{-i\left(\sum_{j=1}^{d} \gamma_{j} \tau_{j}\right) A_{d+1}} A^{\gamma}=: \sum_{|\gamma| \leq N} c_{\gamma} e^{-i \tau_{\gamma} A_{d+1}} A^{\gamma}
$$


so that

$$
\begin{aligned}
\Theta p\left(M_{1}, \ldots, M_{d}\right) & =\sum_{|\gamma| \leq N} c_{\gamma} \Theta e^{-i \tau_{\gamma} A_{d+1}} A^{\gamma} \\
& =\sum_{|\gamma| \leq N} c_{\gamma}\left[S_{\gamma, \tau_{\gamma}}^{*} \otimes \mathrm{Id}\right] \Theta \\
& =\left(\left[\sum_{|\gamma| \leq N} c_{\gamma} S_{\gamma, \tau_{\gamma}}^{*}\right] \otimes \mathrm{Id}\right) \Theta .
\end{aligned}
$$

Letting $m_{j}$ be the multiplier on $D A$ given by $m_{j}\left(\zeta, \zeta_{d+1}\right)=\zeta_{j} e^{-i \tau_{j} \zeta_{d+1}}$, $j=1, \ldots, d$, we have

$$
\begin{aligned}
\left\|p\left(M_{1}, \ldots, M_{d}\right)(v)\right\|_{\mathcal{H}} & =\left\|\Theta p\left(M_{1}, \ldots, M_{d}\right)(v)\right\|_{\mathcal{L}^{2}(\Delta)} \\
& =\left\|\left(\left[\sum_{|\gamma| \leq N} c_{\gamma} S_{\gamma, \tau_{\gamma}}^{*}\right] \otimes \operatorname{Id}\right) \Theta(v)\right\|_{\mathcal{L}^{2}(\Delta)} \\
& \leq\left\|\left[\sum_{|\gamma| \leq N} c_{\gamma} S_{\gamma, \tau_{\gamma}}^{*}\right] \otimes \operatorname{Id}\right\|_{\mathcal{B}\left(\mathcal{L}^{2}(\Delta)\right)}\|\Theta(v)\|_{\mathcal{L}^{2}(\Delta)} \\
& =\left\|\sum_{|\gamma| \leq N} c_{\gamma} S_{\gamma, \tau_{\gamma}}^{*}\right\|_{\mathcal{B}\left(L^{2}\left(\mathbb{N}_{0} \times \mathbb{R}_{-}, d \mu\right)\right)}\|v\|_{\mathcal{H}},
\end{aligned}
$$

so that

$$
\begin{aligned}
\left\|p\left(M_{1}, \ldots, M_{d}\right)\right\|_{\mathcal{B}(\mathcal{H})} & \leq\left\|\sum_{|\gamma| \leq N} c_{\gamma} S_{\gamma, \tau_{\gamma}}^{*}\right\|_{\mathcal{B}\left(L^{2}\left(\mathbb{N}_{0} \times \mathbb{R}_{-}, d \mu\right)\right)} \\
& =\left\|\sum_{|\gamma| \leq N} \overline{c_{\gamma}} S_{\gamma, \tau_{\gamma}}\right\|_{\mathcal{B}\left(L^{2}\left(\mathbb{N}_{0} \times \mathbb{R}_{-}, d \mu\right)\right)} \\
& =\left\|\sum_{|\gamma| \leq N} c_{\gamma} \zeta^{\gamma} e^{-i \tau_{\gamma} \zeta_{d+1}}\right\|_{\mathcal{M}(D A)} \\
& =\left\|p\left(m_{1}, \ldots, m_{d}\right)\right\|_{\mathcal{M}(D A)} .
\end{aligned}
$$

In the general case let $\left(A_{1}, \ldots A_{d+1}\right)$ be Siegel-dissipative. Then, if we replace $A_{d+1}$ by $A_{d+1}+i \varepsilon$ Id we get a strongly Siegel-dissipative tuple of operators. Applying the von Neumann type inequality we have

$$
\left\|p\left(e^{\varepsilon \tau_{1}} M_{1}, \ldots, e^{\varepsilon \tau_{k}} M_{d}\right)\right\|_{\mathcal{B}(\mathcal{H})} \leq\left\|p\left(m_{1}, \ldots, m_{d}\right)\right\|_{\mathcal{M}(D A)},
$$

However, the right hand side of the inequality does not depend on $\varepsilon$ and the left hand side converges in the operator norm as $\varepsilon \rightarrow 0^{+}$. In fact, it suffices to prove the convergence for each term of the polynomial separately; we have

$$
\left\|e^{-i \tau A_{d+1}} e^{\tau \varepsilon}-e^{-i \tau A_{d+1}}\right\|=\left\|e^{-i \tau A_{d+1}}\right\|\left(1-e^{\tau \varepsilon}\right) \leq\left(1-e^{\tau \varepsilon}\right) \stackrel{\tau \nearrow 0}{\longrightarrow} 0 .
$$

Therefore, we can pass to the limit and obtain the desired inequality.

\section{Acknowledgements}

We would like to thank the anonymous referee for his/her helpful comments. 
Funding Open access funding provided by Alma Mater Studiorum - Università di Bologna within the CRUI-CARE Agreement.

Open Access. This article is licensed under a Creative Commons Attribution 4.0 International License, which permits use, sharing, adaptation, distribution and reproduction in any medium or format, as long as you give appropriate credit to the original author(s) and the source, provide a link to the Creative Commons licence, and indicate if changes were made. The images or other third party material in this article are included in the article's Creative Commons licence, unless indicated otherwise in a credit line to the material. If material is not included in the article's Creative Commons licence and your intended use is not permitted by statutory regulation or exceeds the permitted use, you will need to obtain permission directly from the copyright holder. To view a copy of this licence, visit http:// creativecommons.org/licenses/by/4.0/.

Publisher's Note Springer Nature remains neutral with regard to jurisdictional claims in published maps and institutional affiliations.

\section{References}

[1] Agler, J., McCarthy, J.E., Kramer, L.E.: Pick Interpolation and Hilbert Function Spaces, vol. 44. American Mathematical Society, Providence (2002)

[2] Ambrozie, C.-G., Engliš, M., Müller, V.: Operator tuples and analytic models over general domains in $\mathbb{C}^{n}$. J. Operator Theory 47(2), 287-302 (2002)

[3] Arcozzi, N., Levi, M.: On a class of shift-invariant subspaces of the DruryArveson space. Concr. Oper. 5(1), 1-8 (2018)

[4] Arcozzi, N., Monguzzi, A., Peloso, M.M., Salvatori, M.: Paley-Wiener theorems on the Siegel upper half-space. J. Fourier Anal. Appl. 25(4), 1958-1986 (2019)

[5] Arcozzi, N., Rochberg, R., Sawyer, E.: Carleson measures for the DruryArveson Hardy space and other Besov-Sobolev spaces on complex balls. Adv. Math. 218(4), 1107-1180 (2008)

[6] Arcozzi, N., Rochberg, R., Sawyer, E.: Two variations on the Drury-Arveson space. In: Mashreghi, J., Ransford, T. (eds.) Hilbert Spaces of Analytic Functions, pp. 41-58. American Mathamatical Society, Providence (2010)

[7] Arveson, W.: Subalgebras of $C^{*}$-algebras III. Multivariable operator theory. Acta Math. 181(2), 159-228 (1998)

[8] Calzi, M., Peloso, M.M.: Holomorphic function spaces on homogeneous Siegel domains. Diss. Math. 563, 1-168 (2021)

[9] Drury, S.W.: A generalization of von Neumann's inequality to the complex ball. Proc. Amer. Math. Soc. 68(3), 300-304 (1978)

[10] Fang, Q., Xia, J.: On the problem of characterizing multipliers for the DruryArveson space. Indiana Univ. Math. J. 64, 663-696 (2015)

[11] Folland, G.B.: Harmonic Analysis in Phase Space: Annals of Mathematics Studies, vol. 122. Princeton University Press, Princeton (1989)

[12] Lax, P.D.: Translation invariant spaces. Acta Math. 101, 163-178 (1959)

[13] Lax, P.D.: Functional Analysis. Wiley, New York (2002) 
[14] Ogden, R.D., Vági, S.: Harmonic analysis of a nilpotent group and function theory of Siegel domains of type II. Adv. Math. 33(1), 31-92 (1979)

[15] Ortega, J.M., Fàbrega, J.: Pointwise multipliers and decomposition theorems in analytic Besov spaces. Math. Z. 235(1), 53-81 (2000)

[16] Richter, S., Sunkes, J.: Hankel operators, invariant subspaces, and cyclic vectors in the Drury-Arveson space. Proc. Amer. Math. Soc. 144(6), 2575-2586 (2016)

[17] Stein, E. M.: Harmonic analysis: real-variable methods, orthogonality, and oscillatory integrals, volume 43 of Princeton Mathematical Series. Princeton University Press, Princeton, NJ, 1993. With the assistance of Timothy S. Murphy, Monographs in Harmonic Analysis, III

[18] Tchoundja, E.: Carleson measures for Hardy-Sobolev spaces. Complex Var. Ellip. Equ. 53(11), 1033-1046 (2008)

[19] Tchoundja, E.: Carleson measures for the generalized Bergman spaces via a T(1)-type theorem. Arkiv för Matematik 46(2), 377-406 (2008)

[20] Thangavelu, S.: Harmonic analysis on the Heisenberg group. In: Thangavelu, S. (ed.) Progress in Mathematics, vol. 159. Birkhäuser Boston Inc, Boston (1998)

[21] Volberg, A., Wick, B.D.: Bergman-type singular integral operators and the characterization of Carleson measures for Besov-Sobolev spaces on the complex ball. Am. J. Math. 134(4), 949-992 (2012)

[22] von Neumann, J.: Eine Spektraltheorie für allgemeine Operatoren eines unitären Raumes. Math. Nachr. 4, 258-281 (1951)

[23] Waksman, L.: Harmonic analysis of multiparameter semigroups of contractions. In: Commuting Nonselfadjoint Operators in Hilbert Space. Lecture Notes in Math, vol. 1272, pp. 39-115. Springer, Berlin (1987) . (Translated from the Russian by Tatiana de Branges)

Nicola Arcozzi and Nikolaos Chalmoukis $(\bowtie)$

Dipartimento di Matematica

Alma Mater Studorium - Università di Bologna

Piazza di Porta San Donato 5

40126 Bologna

Italy

e-mail: nikolaos.chalmoukis2@unibo.it

Nicola Arcozzi

e-mail: nicola.arcozzi@unibo.it

Alessandro Monguzzi

Dipartimento di Matematica e Applicazioni

Università degli Studi di Milano-Bicocca

Via R. Cozzi 55

20126 Milano

Italy

e-mail: alessandro.monguzzi@unimib.it 
Marco M. Peloso and Maura Salvatori

Dipartimento di Matematica

Università degli Studi di Milano

Via C. Saldini 50

20133 Milano

Italy

e-mail: marco.peloso@unimi.it

Maura Salvatori

e-mail: maura.salvatori@unimi.it

Received: March 16, 2021.

Revised: September 22, 2021. 